\title{
Adaptive Optics for ELTs with Low-Cost and Lightweight Segmented Deformable Mirrors
}

\author{
Gonçalo Rodrigues ${ }^{\mathrm{a}}$, Renaud Bastaits, and André Preumont \\ Université Libre de Bruxelles, 50 av. F.D. Roosevelt CP165/42, B-1050, Brussels, Belgium
}

\begin{abstract}
This paper examines the possibility of constructing deformable mirrors for adaptive optics with a large number of degrees of freedom from silicon wafers with bimorph piezoelectric actuation. The mirror may be used on its own, or as a segment of a larger mirror. The typical size of one segment is $100-200 \mathrm{~mm}$; the production process relies on silicon wafers and thick film PZT deposition technology; it is able to lead to an actuation pitch of the order of $5 \mathrm{~mm}$, and the manufacturing costs appear to grow only slowly with the number of degrees of freedom in the adaptive optics.
\end{abstract}

\section{Introduction}

Adaptive optics (AO) has revolutionized astronomy by correcting in real-time the aberrations introduced by atmospheric turbulence using deformable mirrors with hundreds of actuators. However, attaining diffraction limited imaging in the visible with the current 8-10 m class telescopes will require deformable mirrors with thousands of degrees of freedom [1] (d.o.f.) and correction of turbulence in the future $30 \mathrm{~m}$ class Extremely Large Telescopes will require up to tens of thousands of degrees of freedom[2,3]. Figure 1, on the left, illustrates the increase in the number of degrees of freedom of correction required for imaging $(S t r e h l=0.5)$ when the diameter of the primary mirror increases. It is based on results from [4].

Scaling up the current wavefront correctors and preserving their actuation pitches will result in deformable mirrors with several meters of diameter constituting very complex mechanisms. Such a high complexity will raise issues on development and fabrication costs as well as reliability and maintenance. Moreover, controlling tens of thousands of coupled degrees of freedom of one deformable mirror with a bandwidth of $100 \mathrm{~Hz}$ will constitute a challenge on its own. The problem will become even more complicated if we consider that the scaling up of current weights will most certainly result in a higher dynamic interaction with the light-weight supporting structures and the other control systems of the telescope.

This paper presents a modular system whereby the AO mirror is built as a segmented mirror with hexagonal segments consisting of Si wafers with PZT bimorph actuation. The PZT thick film is deposited by screen printing, whose cost is to a large extent independent of the actuator areal density, and is well adapted to mass production. The expected advantage of the proposed concept in terms of price per degree-of-freedom of correction is shown on the right of Figure 1, when compared to other technologies of deformable mirrors.

We believe that the quality of the manufacturing process, its good repeatability, spatial resolution and control capability are good indicators of the quality of the concept. A full demonstrator involving 3 segments has been constructed.

\section{Silicon Bimorph Mirrors}

The Fraunhofer Institute for Ceramic Technologies and Systems (IKTS) has developed a method for depositing thick films of PZT materials on silicon substrates [7]. Gold electrodes and PZTs, initially in the state of pastes, are deposited by screen-printing and are then sintered, undergoing a phase change and rigidifying.

\footnotetext{
a e-mail: goncalo.rodrigues@ulb.ac.be
} the original work is properly cited. 

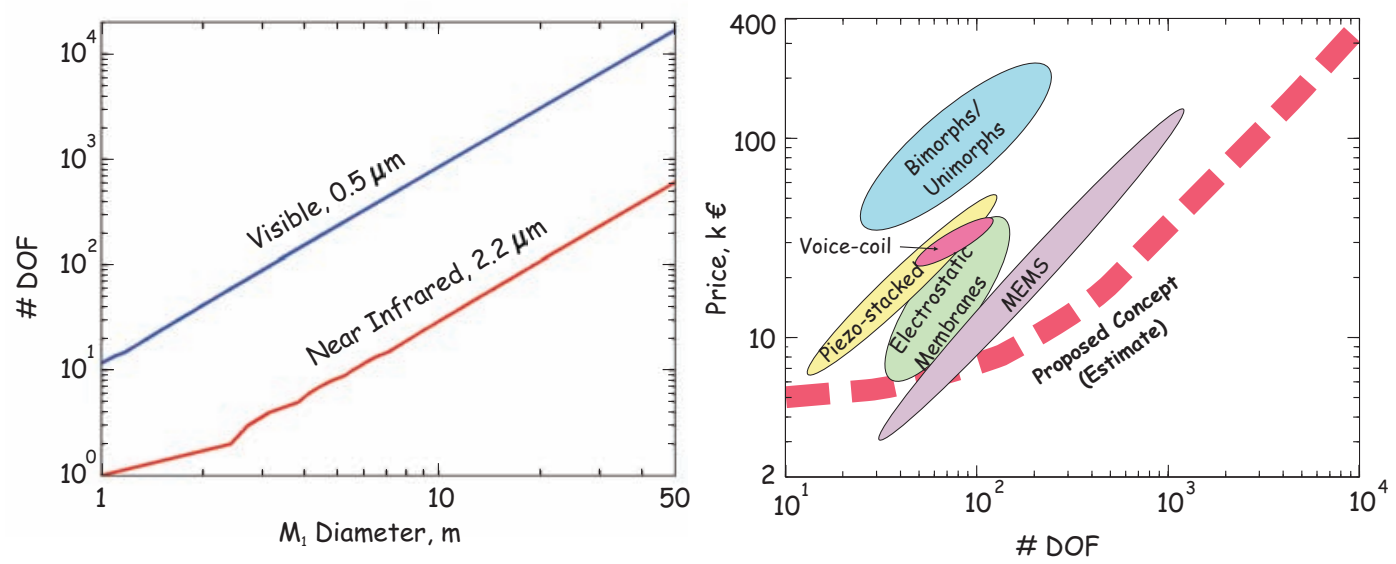

Fig. 1. Left: Evolution of the number of degrees of freedom of the AO with the diameter of the primary mirror M1 in order to achieve diffraction limited imaging, $S$ trehl $=0.5$. Right: Trends in cost (in kEuros/d.o.f.) of existing commercial AO mirrors.
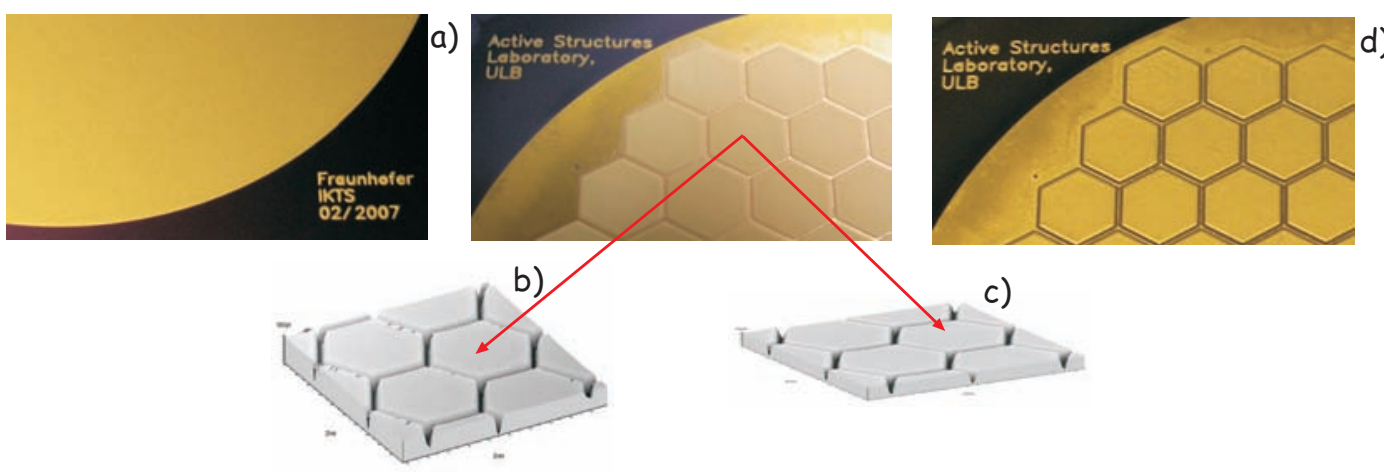

Fig. 2. Silicon bimorph mirror manufacturing steps (a) ground (gold) electrode on the back side of the wafer. (b) 80 micron screen printed PZT array (green) (c) PZT array after sintering. (d) Independent top electrodes (gold).

The steps of the deposition process are depicted in Figure 2. First, a continuous gold film, that serves as the mass electrode common to all the actuators, is screen-printed and sintered. Then, the PZT pastes are screen-printed through a mask with the negative of the actuator patch layout and sintered. Finally, the individual gold electrodes are screen-printed through the same mask on the top of the PZT patches and sintered. The accuracy of the screen-printing process is typically of $\pm 200 \mu \mathrm{m}$

Since the shapes of the PZT patches are completely defined by the form of the mask, once the masks are conceived, their number and their layout does not influence the fabrication time or the price. The same applies to electrical connections if flex-circuits are used.

The PZT patches are $80 \mu \mathrm{m}$ thick, forming a very compact and lightweight laminate. Still, as will be seen below, the amplitudes of deformation are sufficient for applications in adaptive optics. This method of deposition by screen-printing circumvents the common problems that arise, when fabricating and polishing very thin plates of PZT and gluing them to a substrate [8]. Moreover, all the steps of this process can be automated which, given the huge availability of silicon wafers, opens the door to the low-cost mass production of bimorph mirrors.

For the sake of demonstrating the manufacturing technology on the one hand, and verifying the performance on the other hand (stroke, spatial resolution, optical control capability), a bimorph mirror with the layout on the left of Figure 3 was manufactured. 

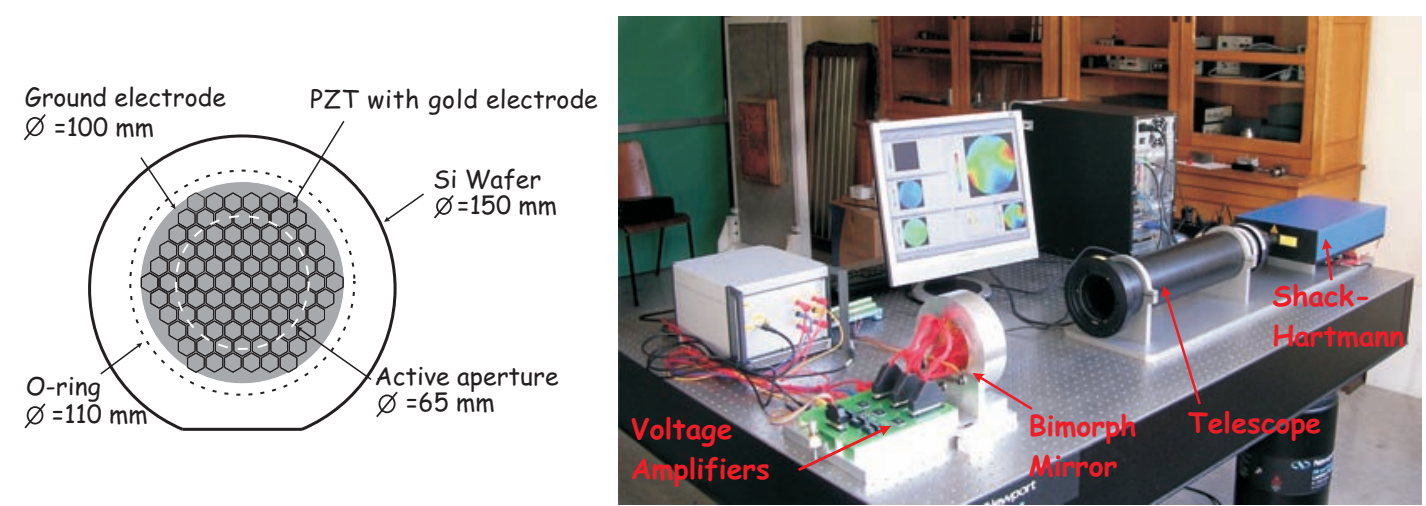

Fig. 3. Left: Layout of the bimorph mirror used in the control experiment. Right: Experimental set-up for the closed loop control experiment. The mirror (in its circular support) is at the front, with the power electronics.

\section{Control Experiment}

An active optics bench has been set-up jointly by the Universite Libre de Bruxelles (ULB) and the Centre Spatial de Liège (CSL) with the aim of assessing the capability of a bimorph mirror produced by screen-printing of performing wavefront correction.

The bimorph mirror appears on the lower left corner of the right of Figure 3 inside its support and is electrically connected to the voltage amplification electronic board.

The measurement of the deformations induced on the mirror is performed by a Shack-Hartmann (S-H) wavefront sensor which appears on the right side of Figure 3.

The closed-loop shape control experiments were carried out statically and consisted of deforming the reflecting surface of the mirror .

Due to the fact that the piezoelectric actuators utilized can mostly sustain positive voltages, the shape control experiments were carried out relative to a reference configuration and consist of 3 steps: (1) Application of an off-set of $80 \mathrm{~V}$ to all the electrodes of the bimorph mirror, and recording the resulting deformation of the mirror as a reference. (2) Application of the set of voltages computed for generation of the shapes of the Zernike modes added of an off-set of $80 \mathrm{~V}$. (3) Subtraction of the reference to the second induced deformation.

Two control experiments have been conducted [9]: (i) a closed-loop experiment using the experimentally calibrated influence functions of the actuators, aimed at evaluating the capability of the system to correct high order optical aberrations, and (ii) an open-loop control experiment using a numerically characterization of the actuator influence functions by finite element analysis [5,6], to evaluate our capability to model the system (it may also be regarded as an estimate of the quality of the manufacturing process).

Figure 4 depicts the modal decomposition of the induced relative deformation when trying to generate the Zernike modes 3 to 35 in closed-loop. In each case, the amplitude of the decomposition is normalized by the amplitude of the generated mode. It can be clearly seen that it was possible to generate each mode without significantly cross-exciting the remaining modes under control.

The voltages computed by purely numerical means have been applied to the experimental setup, and the experimental shape of the mirror was found in excellent agreement with the numerical predictions as patent in Figure 5. This is a good indicator of the quality of the manufacturing process and of the relatively small influence of the hysteresis.

\section{Deformable Segmented Mirrors}

The thick film deposition used in this study, combined with flex-circuits for connectics and low-cost electronics for control, has the potential for being extended to a large number of electrodes with moderate cost. Commercially available Si wafers have a maximum diameter of $300 \mathrm{~mm}$; this means that, 


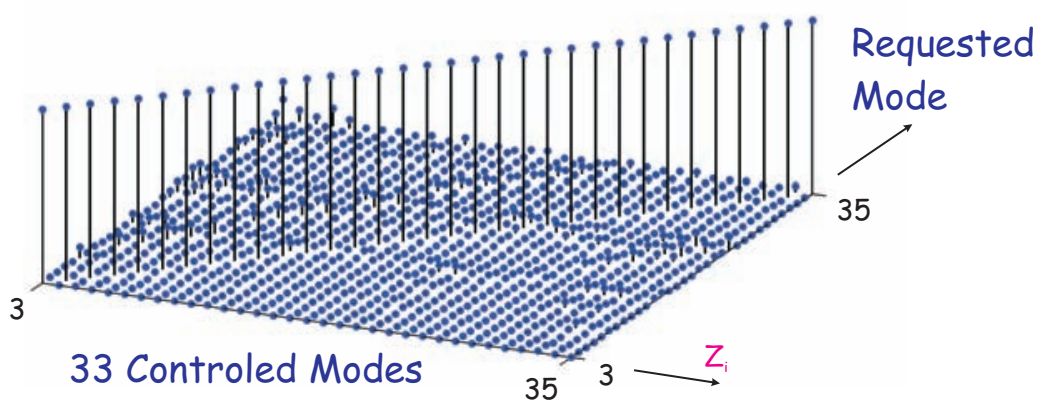

Fig. 4. Closed loop control of the first 33 Zernike modes with curvature (ISO10110-5 convention). Controlled modes vs. requested ones.

Numerical Simulation
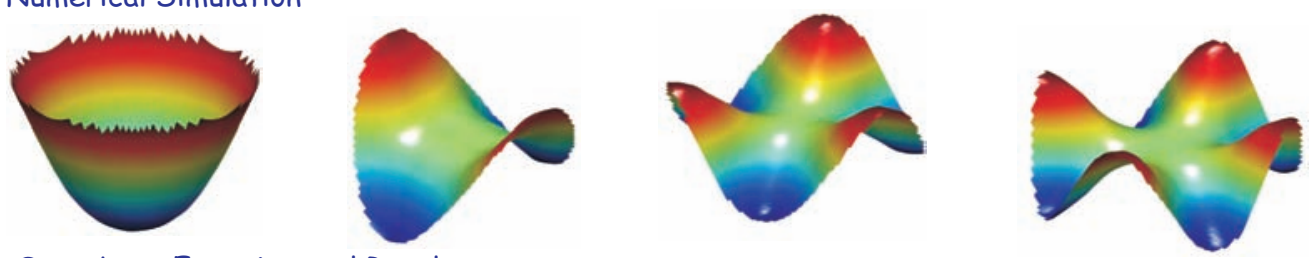

Open-Loop Experimental Results

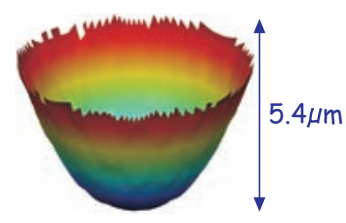

Defocus

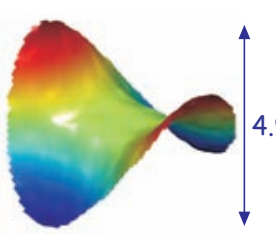

Astigmatism

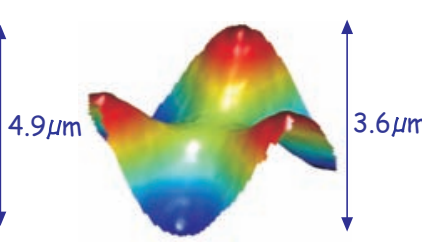

Trefoil

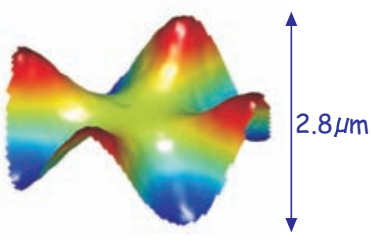

Tetrafoil

Fig. 5. Comparison between numerical simulations and open-loop shape control experiments with numerically computed voltages.

with an actuator pitch of $5 \mathrm{~mm}$, one could possibly fit as much as 2500 electrodes on a single mirror. However, with the modular approach developed here, one can build larger segmented deformable mirrors by modularly assembling hexagonal silicon bimorph mirrors with a honeycomb electrode pattern; the curvature of these mirrors is induced by an array of PZT patches covering the entire back of the segment.

In this modular concept, the distribution of the patches in a honeycomb array confers homogeneity and enables the scaling up. The relative alignment and co-phasing between the segments is achieved by means of linear actuators having a stroke of about $50 \mu \mathrm{m}$ and which actuate each segment in piston, tip and tilt. There are several possible arrangements of the linear actuators to form a kinematic mount, in order to release the in-plane thermal stresses. Assembly and maintenance will privilege configurations in which the segments are completely autonomous and identical. The assembly of the segments with a minimum gap will be easier if all the components of each module lie within the foot-print of the reflecting surface of each segment as illustrated in Figure 6.

In the example of Figure 6, an assembly of 7 segments of $200 \mathrm{~mm}$ diameter, with 127 bimorph patches each, presents more than 520 degrees-of-freedom within the largest circular aperture that can be defined by the assembly and with a pitch of $16 \mathrm{~mm}$. Since the thick film deposition method described in section 2 allows a significantly lower pitch (in the order of $5 \mathrm{~mm}$ ), a significant increase of the resolution of the actuators is possible. The various segments are supported by active kinematic 

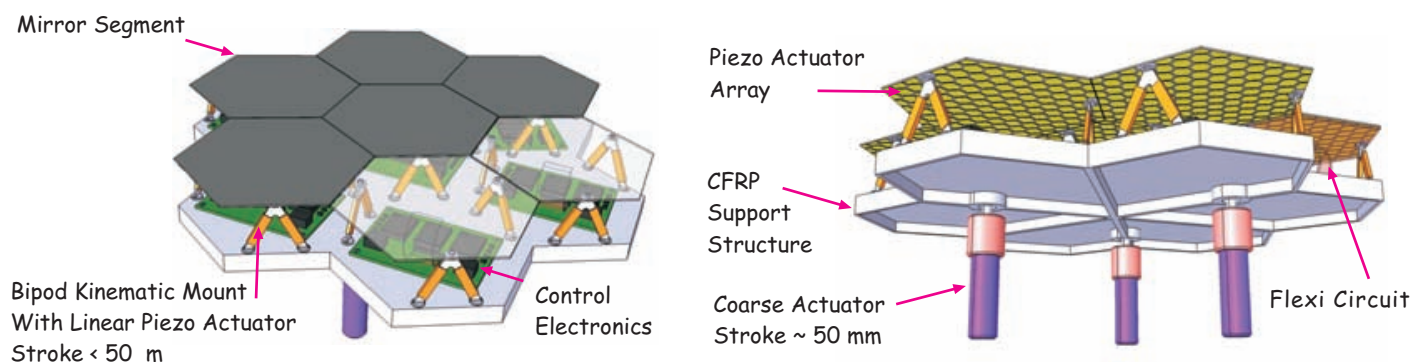

Fig. 6. Segmented bimorph mirror: CAD view of a group of seven segments on its support structure. The size of a segment is between $100 \mathrm{~mm}$ and $260 \mathrm{~mm}$.
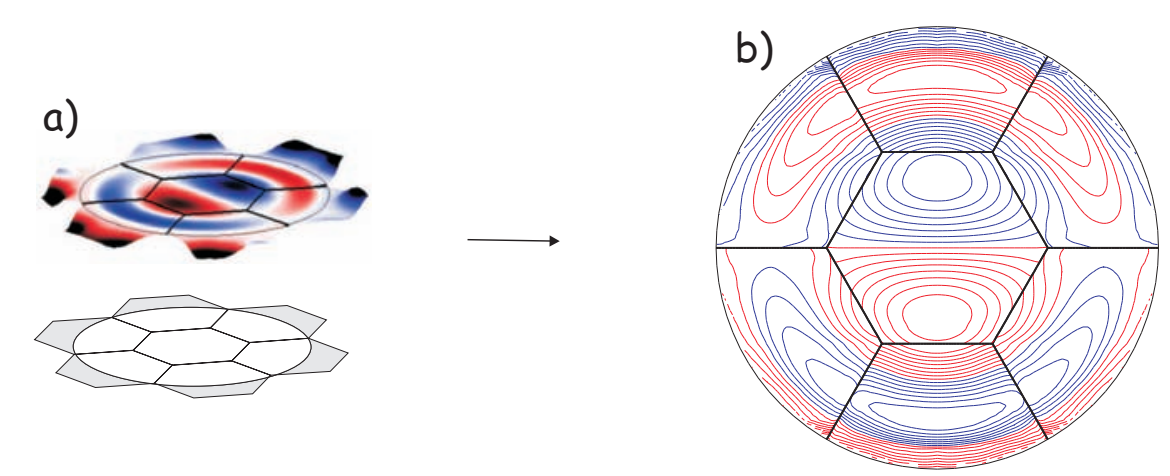

Fig. 7. Numerical simulation of the generation of the mode of 5 th order coma by an assembly of 7 hexagonal bimorph mirrors. (a) with detail of the deformation inside the active aperture (b).

mounts attached to a rigid CFRP support structure, which is itself, if necessary, provided with pistontip-tilt actuators, or mounted on an hexapod (depending on application).

One big advantage of this configuration is that the control of the curvature of various segments is totally decoupled, and identical, leading to the possibility of sensing and correcting in parallel all the portions of the wavefront corresponding to each segment.

Finite element numerical simulations with the material properties of the experiment described in section 3 demonstrate the capability for deforming this assembly of mirrors in a highly continuous manner. As an example, the mode-shape of 5 th order coma was generated inside a $200 \mathrm{~mm}$ aperture with the amplitude required for correcting atmospheric turbulence in the visible in a VLT class telescope, i.e. $46 \mathrm{~nm}$ RMS. The capability of the assembly to generate such a deformation is illustrated in Figure 7 for an assembly of 7 segments with 133 bimorph and 3 linear actuators each, totalizing approximately 550 actuators within the inscribed aperture. The mirror approximates this mode of 5 th order coma with an error of $0.61 \mathrm{~nm}$ RMS.

\section{Demonstrator of Concept}

A prototype formed of 3 segments was assembled for experimentally demonstrating this concept and is illustrated in Figure $8 \mathrm{~b}$ ). Each segment consists of a polished silicon wafer onto which were screenprinted 97 PZT patches and which was cut to a hexagonal boundary. The segments are connected to the voltage amplifiers through flex-circuits. The actuation in piston-tip-tilt is performed in 2 levels. The coarse alignment with relation to the wavefront sensor is done manually using micrometric screws. The fine actuation required for the adaptive correction is done with PZT linear actuators.

The assembly is controlled by a total of 300 control channels. The circular aperture that can be formed at the interface of the 3 segments has a diameter of $130 \mathrm{~mm}$ and contains about 126 PZT in-plane actuators. 

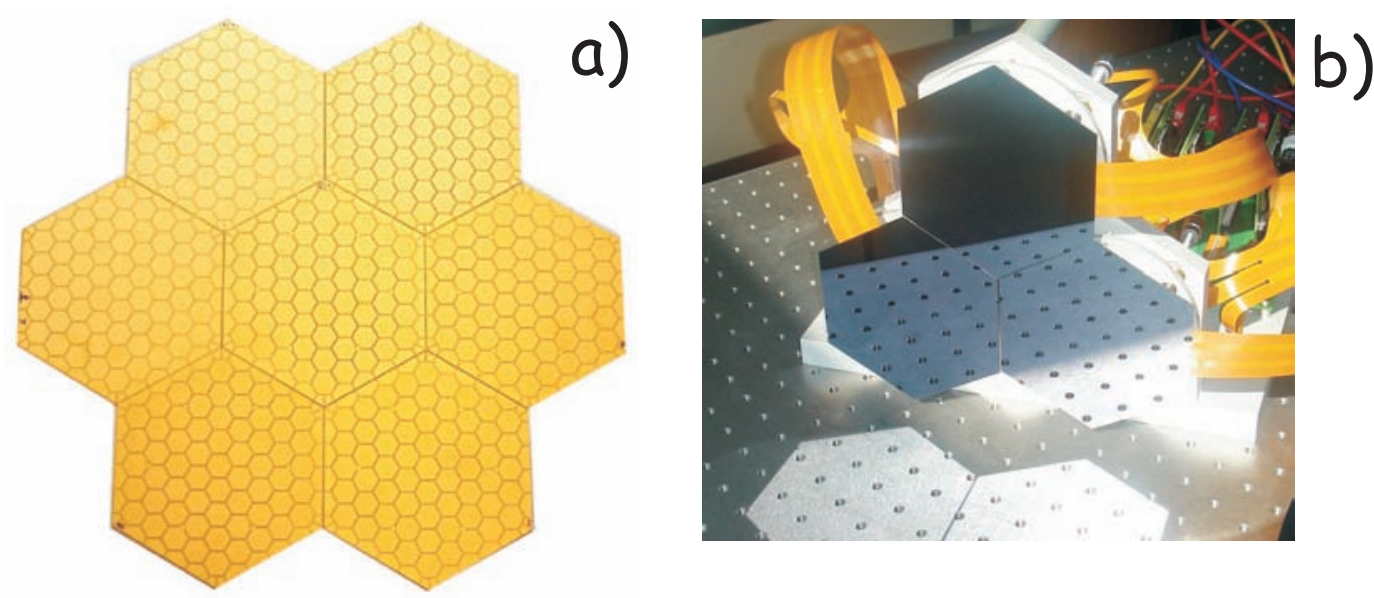

Fig. 8. Hardware of the Segmented Bimorph Mirror. a) Honeycomb of 7 hexagonal screen-printed silicon bimorph-mirrors. b) Front view of the demonstrator of concept formed of 3 segments.

\section{Conclusions}

This paper describes a modular bimorph mirror for adaptive optics applications. The mirror consists of a Silicon wafer covered with an array of thick film PZT deposit with honeycomb electrodes. The mirror may be used on its own, or as a segment of a larger mirror. The current limit on the pitch of the thick film deposition is about $5 \mathrm{~mm}$; thus, with the current size of the wafers, a single mirror may have up to 2500 independent electrodes. The quasi-static behavior of the mirror has been found in very close agreement with numerical finite element predictions. The ability of this mirror to compensate optical aberrations similar to those encountered in adaptive optics has been demonstrated (stroke, spatial resolution, optical modes control capability). Finally, the possibility of constructing large deformable mirrors by assembling segments has been discussed. The manufacturing costs appear to grow only slowly (linearly or less) with the number of degrees of freedom in the AO.

\section{Acknowledgments}

Gonçalo Rodrigues acknowledges Fundação para a Ciência e a Tecnologia, (FCT), Portugal, for his $\mathrm{PhD}$ grant SFRH/BD/21732/2005 and Renaud Bastaits acknowledges Fonds National de la Recherche Scientifique, (FNRS), Belgium, for his FRIA PhD grant FC76554.

\section{References}

1. R. Arsenault et al., Advances in Adaptive Optics II, SPIE 6272, (2006) 62720V

2. N. Hubin, Advancements in Adaptive Optics, SPIE 5490, (2004) 195-206

3. D. Gavel, Adaptive Optics Systems and Technology II, SPIE 4494, (2002) 215-220

4. M. Roggeman and B. Welsh, Imaging through Turbulence (CRC Press, 1996)

5. A. Preumont, Mechatronics - Dynamics of Electromechanical and Piezoelectric Systems (Springer, 2006)

6. V. Piefort, Finite Element Modelling of Piezoelectric Active Structures (Université Libre de Bruxelles, 2001)

7. S. Gebhardt et al., Journal of the European Ceramic Society, 27, (2007) 4177-4180

8. E. Ellis, Low-cost Bimorph Mirrors in Adaptive Optics (Imperial College of Science, Technology and Medicine - University of London, 1999)

9. G. Rodrigues et al., Optical Engineering, 48, (2009) 034001-034001-7 